\title{
A New Mass Model for M31
}

\section{Marc S. Seigar, Aaron J. Barth, and James S. Bullock}

Center for Cosmology, Department of Physics \& Astronomy, University of California, Irvine, 4129 Frederick Reines Hall, Irvine, CA 92697-4575, USA

\begin{abstract}
A new mass model for M31 is presented. The best possible baryonic mass profile for M31 is derived from the 2MASS $K_{s}$-band image and M/L ratios from Bell et al. (2003). The best fitting rotation curve is then found within the context of $\Lambda$ CDM disk galaxy formation. We find that M31 is well-described by a model that includes an adiabatically contracted halo, with an initial NFW concentration, $c=20$. Models without adiabatic contraction fail to reproduce the observed rotation curve at $r>15 \mathrm{kpc}$. The derived halo virial mass is $M_{\mathrm{vir}}=(9.4 \pm 0.8) \times$ $10^{11} M_{\odot}$, consistent with masses derived from other observational methods.
\end{abstract}

\section{Summary}
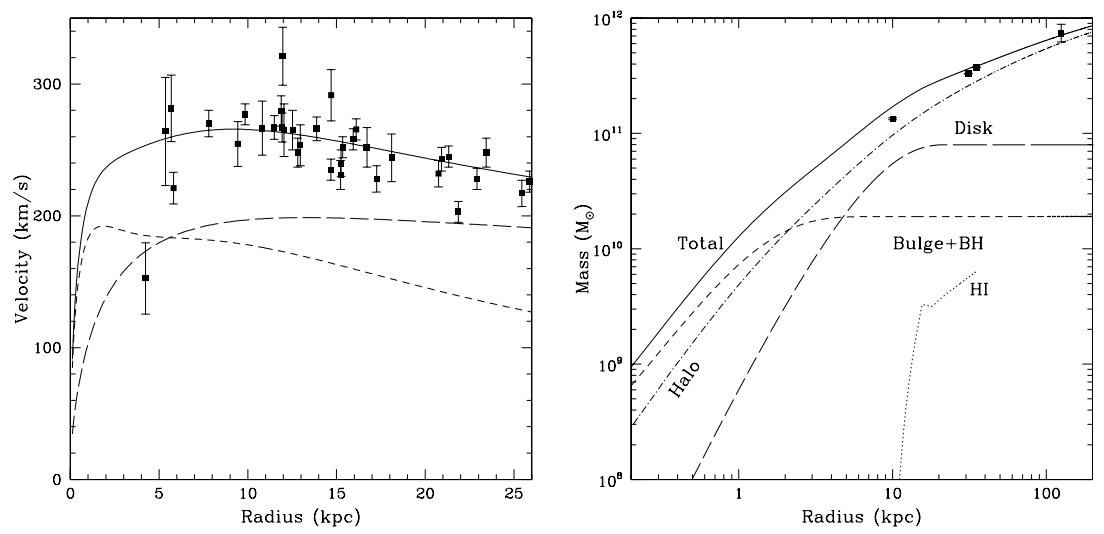

Figure 1. Left: Rotation curve data overlaid with the best fit model (solid line). Right: Enclosed mass as a function of radius.

We use the 2MASS $K_{s}$-band image of M31 and M/L ratios from Bell et al. (2003) to assign masses to the bulge and disk components. A range of allowed dark matter halo masses and density profiles are then explored, using two extreme models for disk galaxy formation, one with adiabatic contraction (AC) and the other without. Figure 1 (left panel) shows observed rotational velocities with the best fitting model rotation curve overlaid. It is consistent with a halo that has undergone AC. The right panel of Figure 1 shows the enclosed mass as a function of radius. The halo virial mass for our model is $(9.4 \pm 0.8) \times 10^{11} M_{\odot}$, similar to the observed mass of $\sim 8 \times 10^{11} M_{\odot}$ from satellite kinematics (Côté et al. 2000). For more details please see Seigar et al. (2006).

\section{References}

Bell, E.F., McIntosh, D.H., Katz, N., \& Weinberg, M.D. 2003, ApJ 585, 117.

Côté, P., Mateo, M., Sargent, W.L.W., \& Olszewski, E.W. 2000, ApJ 537, L91.

Seigar, M.S., Barth, A.J., \& Bullock, J.S. 2006, ApJ submitted. 